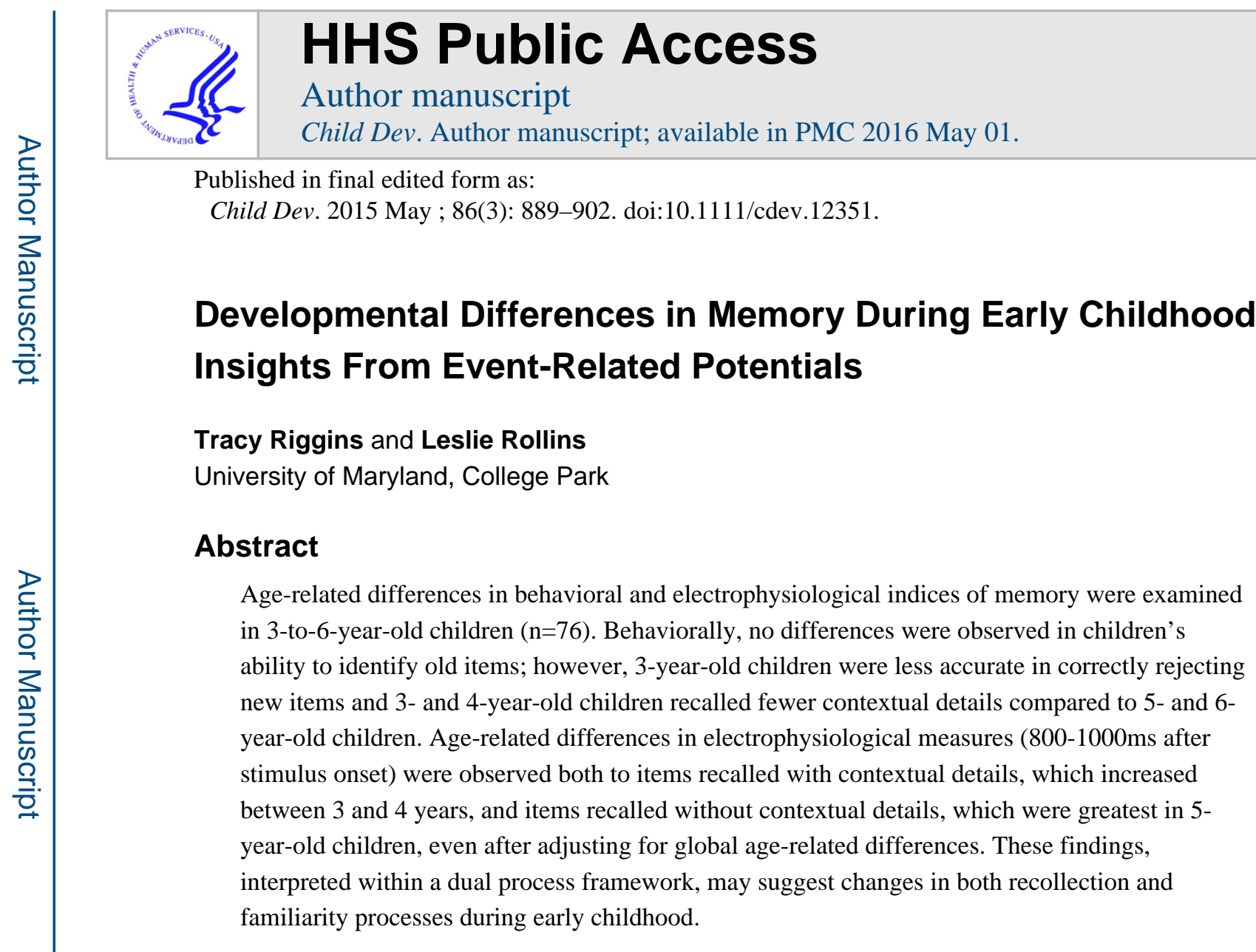

Memory undergoes considerable improvement over the course of childhood. Previous research has documented developmental changes in multiple aspects of memory, including children's ability to recognize previously encountered stimuli as well as their ability to recall contextual details associated with these stimuli across a delay. However, the cognitive and neural mechanisms underlying these changes remain relatively unexplored. In the current study, we sought to address this question by examining age-related differences in memoryrelated neural activity between 3 and 6 years of age.

Previous research suggests that although even very young children are capable of forming memories for items, the ability to recognize these items after increasing delays becomes more reliable and robust over the course of childhood (e.g., Ackerman, 1984; Davidson \& Hoe, 1993; Dirks \& Neisser, 1977; Carroll, Byrne, \& Kirsner, 1985; Cycowicz, Friedman, \& Duff, 2003; Mandler \& Robinson, 1978; Newcombe, Rogoff, \& Kagan, 1977; Parkin \& Streete, 1988, Riggins, 2014). For example, Parkin \& Streete (1988) compared recognition memory for pictures between 3-, 5-, 7-year-old children, and adults after both a 1-hour and 2-week delay. After the 1-hour delay, although all age groups were able to reliably distinguish pictures they had seen before from novel pictures, performance was higher in older age groups. After the 2-week delay, performance in 3-year-olds declined to chance levels and age differences remained between all age groups. Findings such as these have been replicated and extended with longitudinal designs that suggest age-related

Correspondence concerning this article should be sent to Tracy Riggins, Department of Psychology, University of Maryland, College Park, MD 20742. riggins@umd.edu. 
improvements in item memory are relatively linear between 4 and 10 years of age (e.g., Riggins, 2014).

Similarly, children's ability to recall contextual details associated with previously encountered items has also been shown to increase throughout childhood (e.g., Bauer et al., 2012; Cycowicz, Friedman, Snodgrass, \& Duff, 2001; Drummey \& Newcombe, 2002; Ghetti, DeMaster, Yonelinas, \& Bunge, 2010; Hamond \& Fivush, 1991; Riggins, Miller, Bauer, Georgieff, \& Nelson, 2009; Riggins, 2014; Sluzenski, Newcombe, \& Kovacs, 2006). Previous studies have documented that with age children become better able to remember contextual details such as color (Cycowicz et al., 2001; Ghetti et al., 2010), location (Bauer et al., 2012; Sluzenski et al., 2006), temporal order (Riggins et al., 2009), and source (Drummey \& Newcombe, 2002; Riggins, 2014). Consistent with the age-related differences reported in cross-sectional studies, a recent longitudinal study suggested that early childhood may be a period of particularly rapid change in the ability to remember contextual details (see Riggins, 2014 for details).

Despite relative agreement that children's ability to recognize previously encountered items and their details improves across childhood, the cognitive processes and neural mechanisms that underlie these developmental changes remain relatively unexplored, particularly during early childhood. One prominent memory theory asserts that two processes (recollection and familiarity) underlie individual's ability to remember (Yonelinas, 2002). Recollection allows for the retrieval of distinct features associated with the context in which the event was originally encountered, whereas familiarity allows for the global assessment of the strength of the memory trace of the event without contextual features. Although controversy exists surrounding dual processes models of memory (Malmberg, 2008; Squire, Wixted \& Clark, 2007; Wixted \& Mickes, 2010), empirical evidence has accumulated from multiple sources including experimental, computational modeling, neuropsychological, neuroimaging, and individual differences perspectives (see Yonelinas, 2002 for review). Developmental research suggests that recollection and familiarity follow different developmental trajectories in middle childhood and adolescence (e.g., Ghetti \& Angelini, 2008). Data from these studies suggest that recollection develops well into the adolescent years whereas familiarity shows comparatively little, if any, development after 6-8 years of age (Ghetti \& Angelini, 2008, see also, Anooshian, 1999; Anooshian \& Seibert, 1996; Billingsley, Smith \& McAndrews, 2002; Brainerd, Holliday, \& Reyna, 2004; Cycowicz et al., 2003; Czernochowski, Mecklinger, Johansson, Brinkmann, 2005; Drummey \& Newcombe, 2002 Ghetti et al., 2010; Brainerd, Aydin, \& Reyna, 2012; Friedman, de Chastelaine, Nessler, \& Malcom, 2010; Mecklinger, Brunnemann, \& Kipp, 2011). Although research in younger children has documented that both familiarity and recollection contribute to memory in 1- to 3-year-old children (e.g., Rose, Feldman, Jankowski, \& Van Rossem, 2011), the developmental trajectories of these processes and their relative contribution to recognition memory early in life remain unknown (see Brainerd, Reyna, \& Howe, 2009; Newcombe \& Crawley, 2007; Riggins, 2012 for similar arguments) and theoretical debates still exist regarding the nature of memory early in life (e.g., Nelson, 1997; Rovee-Collier, 1997). These controversies remain, in part, as a result of methodological challenges associated with examining memory mechanisms early in life. 
Event-related potentials (ERPs) may be useful in investigating mechanisms of memory in early childhood, as they can provide insight into the brain's response under different memory conditions and allow one to obtain information regarding processes underlying memory that may not be accessible via behavioral measures. For example, ERP studies of memory in adults have yielded considerable insight into the neural networks underlying explicit memory. This research has linked distinct cognitive processes (e.g., recollection and familiarity) to specific ERP effects (e.g., left-parietal old/new effect and FN400, respectively) and these findings have been used to inform theoretical debates regarding the nature of memory (see Friedman \& Johnson, 2000; Rugg \& Curran, 2007 for reviews). Although ERP measures have been used to examine memory processes early in life, few studies have systematically examined age-related changes in these processes or their specificity. Thus, little is known about age-related changes in memory ERP components and the memory processes related to them particularly during early childhood when changes at the behavioral level are well documented.

In terms of recognition memory, studies have shown that differences in neural responses to old and new items (i.e., memory-related effects) can be detected using ERPs across a wide range of ages (6 months to adulthood, e.g., Bauer, Wiebe, Carver, Waters, Nelson, 2003; Carver, Bauer, \& Nelson, 2000; Cycowicz et al., 2001; Marshall, Drummey, Fox, \& Newcombe 2002; Rollins \& Riggins, 2013; Riggins et al., 2009; Riggins, Rollins, \& Graham, 2013; for review of research in infants see de Haan, 2007, in adults see Friedman \& Johnson, 2000; Rugg \& Curran, 2007). However, comparison of age-related differences across these studies is complicated by their use of different memory paradigms and analytic approaches. Thus, it remains unclear to what extent differences between studies examining various age groups reflect developmental differences versus variations in experimental methodologies. Of the few studies that have included multiple age groups, findings suggest that memory-related ERP effects are sensitive to developmental changes. For example, in one of the first studies in early childhood, Marshall and colleagues (2002) compared ERP responses of 4-year-old children $(300-600 \mathrm{~ms}, 600-900 \mathrm{~ms}$, and $900-1500 \mathrm{~ms})$ and adults (450-700 ms and 700-1350ms) generated during the retrieval phase of an intentional picture recognition memory paradigm. In both children and adults, amplitude varied between correctly recognized old items and correctly rejected new items across multiple scalp locations (i.e., frontal, central and parietal leads in both groups), suggesting it was a sensitive measure of item recognition memory at both ages. However, memory-related ERP effects differed between children and adults in terms of timing and distribution across the scalp. Specifically, effects were observed later (i.e., between $900-1500 \mathrm{~ms}$ as opposed to 700-1350ms) and were more localized (i.e., in the right hemisphere at F4, C4, O2, and Pz, as opposed to bilaterally at $\mathrm{F} 3, \mathrm{~F} 4, \mathrm{O} 1, \mathrm{O} 2, \mathrm{Pz}$ ) in children compared to adults, which suggested that these measures can provide information about age-related differences in neural activity supporting memory.

Several ERP studies haven examined age-related improvements in memory for contextual details between school-aged children, adolescents, and adults (e.g., Cycowicz et al., 2003; Czernochowski et al., 2005; Sprondel, Kipp, \& Mecklinger, 2011, see also Friedman et al., 2010). These studies typically utilize source memory tasks, which require participants to remember details associated with individual items (e.g., the color or location or experimental 
run in which items were presented). For example, Sprondel and colleagues (2011) examined changes in ERPs during a continuous recognition memory task in a sample of school-aged children (7-8 years), adolescents, and adults that required participants to discriminate between repeated presentations of object and nonobject stimuli across two experimental runs. As expected, behavioral measures revealed that adolescents and adults were better than children at this task. ERP responses were used to examine item memory (comparing old and new stimuli) and source memory (comparing old non-targets to new stimuli). Results for item memory suggested differences in ERP responses to old and new stimuli both early $(350-450 \mathrm{~ms})$ and late $(650-800 \mathrm{~ms}$ in children and $450-600 \mathrm{~ms}$ in adolescents and adults). However, the distribution of these effects differed between the groups. In the early time window (350-440ms), a reliable early old/new effect was observed for all age groups, however the topographic distribution differed; in children and adolescents the effect was observed over parietal midline sites (Pz), whereas in adults the effect was apparent across all midline leads. In the later time window $(650-800 \mathrm{~ms}$ in children and $450-600 \mathrm{~ms}$ in adolescents and adults), the old/new effect was maximal over parietal sites (i.e., a parietal old / new effect at $\mathrm{Pz}$ ) in children and adults in contrast with adults where it was maximal over right-frontal leads (i.e., F4). Results of the source memory analyses revealed two effects of interest: 1) a parietal non-target old/new effect that was widespread for adolescents (750-900ms, across all leads) and localized in adults $(450-600 \mathrm{~ms}$ at $\mathrm{Cz}$ and $\mathrm{Pz}$ ) but not apparent at all in children $(800-950 \mathrm{~ms})$, as well as 2) a late right-frontal effect in adults only (850-1000ms at F4). Based on these results, authors asserted that developmental changes in behavioral performance resulted from changes in two memory-related ERP effects: the parietal old/new effect (associated with recollection) and the right-frontal old/new effect (associated with postretrieval monitoring, see Friedman \& Johnson, 2000; Rugg \& Curran, 2007 for reviews). Findings such as these illustrate how age-related differences in ERPs may be used to elucidate the processes that underlie improvements in memory.

Only recently have ERP studies been used to investigate processes underlying memory for contextual details in early childhood (e.g., Riggins et al., 2009a; 2009b; Riggins et al., 2013). Similar to studies in older groups, Riggins and colleagues (2013) utilized ERPs to examine processes underlying successful performance on a source memory task in 5-yearold children. In this study, children viewed and interacted with objects in one of two locations. Following this encoding period, ERPs to old and new stimuli were recorded during passive viewing after which children completed behavioral versions of item and source memory tasks to identify which items they had seen before and if they recalled the contextual detail associated with them. Results showed that accuracy on the source memory task (i.e., the ability to recall the contextual detail associated with the items) was related to ERP amplitude. Between $800-1500 \mathrm{~ms}$, amplitude at P3 and P4 to items recalled with correct source information differed from both items recalled with incorrect source information and new items. In addition, activity over left temporal leads (AF7, F3, FC3, C3, CP3, P3) was correlated with behavioral performance, such that greater amplitude differences between source-correct and new items was related to better performance on the source memory task). Together, these finding suggest that activity late in the ERP response may reflect memory processes involved in or associated with memory for contextual details (an interpretation 
consistent with previous literature in infants suggesting activity in this time window is generated by sources within the temporal lobe, see Reynolds \& Richards, 2005). However, this study did not explore age-related changes in the ERP response, and thus the extent to which this effect is present over the course of early childhood remains unknown.

\section{Present Study}

Examination of age-related changes in ERP responses during early childhood may help shed light on developmental improvements in memory during a period when such questions have been difficult to address. As such, the goal of the current study was to examine age-related differences in the neural responses to three theoretically relevant conditions: old items remembered with contextual details (which require recollection processes), old items remembered without contextual details (which can be supported by familiarity alone), and correctly rejected new items (which do not engage familiarity or recollection processes directly). If there are developmental changes in recollection, age-related differences would be apparent in ERP responses to old items remembered with contextual details, as these responses require recollection. If there are developmental changes in familiarity, age-related differences would be apparent in ERP responses to old items remembered without contextual details, as these responses can be supported by familiarity alone. Finally, if there are developmental changes in general cognitive abilities, but these changes are not specific to memory, then age-related differences would be apparent in ERP responses to new items, as these responses do not engage recollection or familiarity.

Based on previous studies with children in this age range (Marshall et al., 2002; Riggins et al., 2013), we hypothesized that two components of interest would be apparent in the ERP response: the Nc (a negative deflection 400-600ms post stimulus onset) and the positive slow wave (PSW, a distributed positivity occurring $800-1500 \mathrm{~ms}$ post stimulus onset). These components have been previously identified in developmental ERP memory literature (e.g., Bauer et al., 2003; Marshall et al., 2002; Riggins et al., 2009; Riggins et al., 2013; Sprondel et al., 2011, for review see de Haan, 2007), however knowledge remains limited as to 1) their engagement in tasks that differentially rely on recollection and familiarity (cf. Riggins et al., 2013) and 2) how these components change as a function of age.

As reviewed above, in the present study 3- to 6-year-old children were examined because this period represents a time during which memory has been shown to undergo significant improvements (e.g., Riggins, 2014). Previous behavioral research in children aged 4 years and older (e.g., Ghetti \& Angelini, 2008; Lloyd, Doydum, \& Newcombe, 2009; Sluzenski et al., 2006) suggests differences will be present in the "recollection" condition (i.e., items recalled with contextual details). Based on these documented changes at the behavioral level, we hypothesized that age-related differences in memory processes would be observed at the neural level. These changes may be reflected either in the magnitude of the ERP effects or in the pattern/distribution of the ERP effects. However, because this is a period during which little is known about the processes underlying these changes, specific predictions regarding the direction of the effect or its distribution across the scalp were not warranted (Brainerd et al., 2009; Newcombe \& Crawley, 2007; Riggins, 2012). In contrast, to the best of our knowledge, no previous study during early childhood has examined age- 
related differences in "familiarity" (or the processing of stimuli which children judge to be familiar but that lack evidence of contextual details). Thus, no predictions were made regarding results from the examination of these responses.

\section{Method}

Participants

Participants were drawn from a larger study examining memory development in early childhood. A total of 76 children provided both behavioral and ERP data for the three conditions that are relevant to the present report: 183 -year-old children (mean age $=3.28$ years, $S D=.13,6$ females, 12 males), 184 -year-old children (mean age $=4.28$ years, $S D=$. 15, 11 females, 7 males), 23 5-year-old children (mean age $=5.28$ years, $S D=.13,11$ females, 12 males), and 17 6-year-old children (mean age $=6.26$ years, $S D=.18,10$ females, 7 males). All participants were born full-term, heard English at least $80 \%$ of the day, and had no known psychopathology. Of the sample, $63 \%$ of participants reported being White/Caucasian, 17\% Black/African American, 5\% Asian, 9\% multiracial, and 5\% did not wish to disclose this information.

\section{Materials}

Behavioral stimuli consisted of 81 commercially available items. Fifty-four of the items were presented at both the encoding and retrieval visits; an additional 27 items were presented as novel items at the retrieval visit (see Procedure below). Each item was randomly associated with a novel action (hug it, put it on your head, beat on it like a drum). Then, items were randomly divided into 9 sets of 9 items. Each action was represented 3 times per set. ERP stimuli consisted of 4.5 " $\times 8$ " digital color photographs of the behavioral stimuli presented at the most canonical viewing angle.

\section{Procedure}

The University Institutional Review Board approved all procedures prior to data collection. Participants were recruited from a database maintained by the University's Infant and Child Studies program.

The procedures used in the present study were modeled after procedures in Riggins et al., (2013). All children made two visits to the lab, 1-2 days apart (mean delay $=1.33$ days, $S D$ $=.47$; there were no difference between the age groups in delay, $p=.74$ ). At the first visit, all study procedures were described. Parents provided written informed consent and children provided verbal assent. Following the consent process, each child was shown 54 study items in 1 of 2 different locations. Location and item set order were counterbalanced between participants; items within sets were presented randomly. Locations consisted of two childfriendly rooms decorated to be visually interesting but distinct from each other. Each room had a stuffed "character" associated with it to increase the salience of the context. Inside each room, children were shown each item individually and instructed to interact with the item. This served as a baseline assessment of what actions children would typically perform. Following baseline for each item, the experimenter modeled the action associated with the object and requested that the child imitate the action. This manipulation was included 
because action imitation has been shown to support subsequent memory performance beyond that of event observation (Lukowski et al., 2005). A 5 to 10 minute delay was introduced between locations to temporally separate the encoding of items in each context. At the end of the session, children received a small toy for their participation.

At the second laboratory visit, children participated in an ERP paradigm and a behavioral recall session. The fixed presentation order of the ERP and behavioral task was used because behavioral testing could influence the ERP response (e.g., by giving children experience with the novel stimuli). Prior research has demonstrated no effect of ERP exposure on subsequent recall performance (Bauer et al., 2003; Carver et al., 2000). Children were first fitted with an appropriate size electrode cap and seated in front of a computer screen. Children were not given instructions but were informed that they would observe pictures of items they interacted with at the previous session and pictures of new items. In order to remind children of the previous session in general, pictures of the "characters" from each room were displayed. However, pictures of the two rooms were not displayed and children were not reminded of the actions. Children were instructed to remain as still as possible and no overt behavioral response was required, which served to minimize movement artifact (DeBoer, Scott, \& Nelson, 2005). After data collection, ERP trials were back-sorted based upon subsequent behavioral performance in the source memory paradigm (see below).

EEG was recorded from 64 scalp locations, left and right mastoids, two vertical electrooculogram (EOG) and two horizontal EOG channels using active $\mathrm{Ag}-\mathrm{AgCl}$ electrodes (BioSemi Active 2) at a sampling rate of $512 \mathrm{~Hz}$. Stimuli were presented on the screen for $500 \mathrm{~ms}$, followed by a fixation cross which varied in duration from 1250 to $1700 \mathrm{~ms}$. Children viewed the stimuli during three separate blocks. Each block consisted of random presentation of the 54 previously seen (target items) and 27 new (distracter) items, for a total of 243 ERP trials. Three blocks were used because 1) during pilot testing we found this strategy was more effective than increasing the number of to-be-remembered items, which significantly decreased memory performance, and 2) previous research suggests such repeated presentation does not significantly alter condition differences in the ERP response (Riggins et al., 2013). (Note: To determine how the 3 presentations impacted the ERP response for old and new items respectively, we conducted a separate analysis examining average amplitude to items in the first, second, and third blocks. Specifically, we used a 3 Block (first, second, third) $\times 2$ Condition (old, new) $\times 3$ Coronal Plane (frontal, central, parietal) $\times 3$ Sagittal Plane (left, midline, right) RMANOVA including 9 leads (F3, $\mathrm{Fz}, \mathrm{F} 4, \mathrm{C} 3, \mathrm{Cz}, \mathrm{C} 4, \mathrm{P} 3, \mathrm{Pz}, \mathrm{P} 4)$ to examine activity in 2 Time Windows in which we observed significant differences between old and new stimuli: $350-500 \mathrm{~ms}$ and $800-1100 \mathrm{~ms}$. Ten participants yielded useable data for this analysis. Results revealed a marginal main effect of Block for the 350-500ms Window, $F(2,18)=3.04, p=.07, \eta^{2}=.25$. Average amplitude decreased across blocks (Block $1=-5.54 \mu \mathrm{V}$, Block $2=-4.67 \mu \mathrm{V}$, Block $3=$ $-4.43 \mu \mathrm{V})$. However, this effect did not differ between the Conditions. No effect of Block was observed for the 800-1100 Time Window.)

Following ERP data collection, the electrode cap was removed and children participated in the retrieval portion of the memory paradigm. All 81 behavioral stimuli were presented individually to the children who were asked whether they had seen the item on their first 
visit or not. If an item was identified as having been seen (i.e., "old"), children were asked 1) what action (out of 3) was paired with the item during the previous visit and 2) which location (out of 2) they had encountered it in. If the children reported that they had not seen the item on the previous visit (i.e., "new"), they were asked to place the item into a "new item" bin (i.e., questions regarding action and location were not asked). In addition to ageappropriate instructions, five training trials were administered to ensure all children understood the task. At the end of the session, children received a small toy and a certificate for their participation.

\section{Data Reduction and Analytic Approach}

Behavioral data-For the memory task, correctly identified target items are referred to as "old" and correctly identified distracter items are referred to as "new." Items correctly identified as old for which both action and location details were correctly recalled are referred to as " 2 details correct," items correctly identified as old for which one detail was correctly recalled (action or location) are referred to as "1 detail correct," and items correctly identified as old for which neither action nor location was correctly recalled are referred to as " 0 details correct." Incorrectly identified distracter items that were judged as old are referred to as "false alarms." The sensitivity index ( $\left.d^{\prime}\right)$ was computed as the discrepancy in standard deviation units between the proportion of old and new pictures to which the participant correctly responded "yes" (Macmillan \& Creelman, 2005). When the assumption of the homogeneity of variance was violated for any dependent measure (as indicated by Levine's statistic) the Welch F-ratio and Dunnett's T3 posthoc tests are reported.

Electrophysiological data-Electrophysiological data were re-referenced offline to an average reference using Brain Electrical Source Analysis (BESA) software (MEGIS Software $\mathrm{GmbH}$, Gräfelfing, Germany). Missing data from individual channels was interpolated for a maximum of $10 \%$ of bad channels (i.e., 8 per participant; see DeBoer et al., 2005). Consistent with previous ERP studies in children, ocular artifacts were corrected by applying the Ille, Berg, \& Scherg (2002) algorithm (Cycowicz et al., 2003; Marshall et al., 2002; Riggins et al., 2013). Data were high pass filtered at $0.1 \mathrm{~Hz}$ and low pass filtered at $80 \mathrm{~Hz}$. A $30 \mathrm{~Hz}$ low pass filter was used for illustrations. Movement related artifacts were hand-edited and rejected prior to averaging. Trials were epoched with a $100 \mathrm{~ms}$ baseline and continued for $1500 \mathrm{~ms}$. ERPs were averaged into separate conditions based on behavioral performance as described above (i.e., items recalled with 2 details, items recalled with 0 contextual details, and new items). Participants with fewer than 10 trials per condition were excluded from analysis (DeBoer et al., 2005). Trial numbers (mean, standard deviation, and range) for each condition were as follows: 2-details $(M=25.70, S D=11.30,10-57)$, 0 details $($ mean $=16.88, S D=6.27,10-44)$, New $($ mean $=38.89, S D=14.13,10-68)$. There were significant differences in the number of trials between Age Groups for 2-detail trials, $F(3,72)=9.17, p<.001$, and a marginal difference between Age Groups for new trials, $F(3$, $72)=2.70, p=.052$. For 2-detail trials, 3-year-olds had fewer trials than all other age groups, and for new trials, 3-year-olds had fewer trials than 6-year-olds. Average amplitude served as the dependent measure for all analyses because it is relatively unbiased by differences in the number of trials between conditions (Luck, 2005). In addition, there was no correlation between the number of trials and average amplitude at the vertex for any of 
the 3 conditions (2-details, 0 -details, New) for either early (Nc), $p$ s > .11, or late (PSW) components, $p s>.15$.

The resulting waveforms contained two components closely resembling the $\mathrm{Nc}$ and the positive slow wave (PSW), which have been previously identified in developmental ERP memory literature (e.g., Bauer et al., 2003; Marshall et al., 2002; Riggins et al., 2009; Riggins et al., 2013; for review see de Haan, 2007). Based on these previous studies, visual inspection, and preliminary analyses, two windows were selected for ERP analysis: $350-500 \mathrm{~ms}$ and $800-1000 \mathrm{~ms}$ corresponding to the $\mathrm{Nc}$ and PSW respectively.

Repeated measures ANOVAs were used to examine differences between conditions in 2 different time windows (350-500ms, and 800-1000ms) using a 4 Age Group (3, 4, 5, 6 years) $\times 3$ Condition (2-details, 0 -details, new) $\times 3$ Coronal Plane (frontal, central, parietal) $\times 3$ Sagittal Plane (left, midline, right) and included the following leads: F5, Fz, F6, C5, Cz, $\mathrm{C} 6, \mathrm{P} 5, \mathrm{Pz}, \mathrm{P} 6$. Greenhouse-Geisser corrections for non-sphericity were used. Only main effects and interactions with Condition are reported. When Condition effects differed as a function of Age Group (as indicated by a significant Condition $\times$ Age Group interaction) follow-up analyses were first conducted for each Age Group separately to investigate whether condition effects were apparent in each group and then by Condition to investigate patterns of age-related differences in ERP activity to each of the conditions. In the latter, in order to ensure any observed effects were due to memory processes (as opposed to general age-related effects), difference waves were computed between each memory condition (2details, 0-details) and new items. Measures resulting from this difference wave analysis are referred to as 'adjusted' amplitudes and were subjected to the same omnibus RMANOVA as described above.

\section{Results}

\section{Behavior}

Analysis of behavioral performance suggested there was a significant difference between the Age Groups' ability to discriminate old and new items (i.e., d'), $F(3,72)=5.05, p<.01$., $\eta^{2}$ $=.17$. Follow-up tests showed 3 -year-old children had marginally lower d' values compared to 4-, 5-, and 6-year-old children, who did not differ from each other (see Table 1). Inspection of correctly recognized old items (hits) and false alarms separately (see Lloyd, et al., 2009, for justification) revealed that this effect was driven by differences in the children's ability to correctly reject new items. Three-year-old children made more false alarms (i.e., correctly rejected fewer stimuli) than older children, $F(3,34.98)=3.23, p<.05$, $\eta^{2}=.12$, see Table 1 . In contrast, differences between the Age Groups' ability to correctly identify old items failed to reach conventional levels of significance $(p=.08)$.

In addition, the number of contextual details children were able to recall increased as a function of age, $F(3,72)=10.06, p<.01, \eta^{2}=.30$. Specifically, the proportion of items children were able to recall with 2 contextual details increased with age, $F(3,72)=6.71, p$ $<.001, \eta^{2}=.22$. Three-year-old children recalled significantly fewer details than 4-yearolds, who recalled marginally fewer details than 5-year-olds. However, the number of details recalled by 5 - and 6-year-old children did not differ. 
It is important to note that despite these differences, all groups were able to reliably perform the task; even the youngest group (3-year-olds) recalled significantly more items with 2 contextual details than would be expected by chance, $t(17)=4.63, p<.001$, Cohen's $d=$ 2.25. The proportion of items children identified as old but for which they were unable to recall any contextual details (i.e., 0 details) decreased with age, $F(3,72)=10.59, p<.001, \eta^{2}$ $=.31$. The proportion of items children were able to recall with 1 contextual detail did not differ as a function of age, $p=.74$; however, this result is not particularly informative given that there was no difference between the age groups in the ability to identify old items and inclusion in one contextual detail condition precluded inclusion in the other conditions.

\section{Event-Related Potentials}

Grand average waveforms for each Age Group are presented in Figure 1. Results suggested that between $800-1000 \mathrm{~ms}$ differences between conditions were apparent across the age groups, but that the pattern of differences changed with age, and, ultimately resulted in 6year-old children showing the most differentiated patterns of activity. Specifically, in 3-, 4-, and 6-year-old children, ERP amplitude to new items was greater than amplitude to old items recalled without contextual details, with amplitude to old items recalled with contextual details in between. However, the distribution of this effect changed with age as it was observed over frontal and parietal leads in 3-year-old children but over left hemisphere leads in 4- and 6-year-old children. In 5- and 6-year-old children, amplitude to old items recalled without contextual details was greater than amplitude to old items recalled with contextual details, with amplitude to new items in between. Again the distribution of this effect differed between the age groups as it was observed over central leads in 5-year-old children and over the parietal midline lead in 6-year-old children. Finally, 6-year-old children also showed greater amplitude to items recalled with 2 details compared to items recalled with 0 details at frontal midline and right central leads. To summarize, although all age groups showed differences between conditions, the direction of effects and their distributions differed as a function of age. Supporting analyses are below.

Analysis of the early time window (350-500ms) using a 4 Age Group (3, 4, 5, 6 years) $\times 3$ Condition (2-details, 0-details, new) $\times 3$ Coronal Plane (frontal, central, parietal) $\times 3$ Sagittal Plane (left, midline, right) RMANOVA revealed no significant main effects or interactions with Condition, $p s>.15$. Analysis of the later time window (800-1000ms) using a 4 Age Group $(3,4,5,6$ years $) \times 3$ Condition (2-details, 0 -details, new) $\times 3$ Coronal Plane (frontal, central, parietal) $\times 3$ Sagittal Plane (left, midline, right) RMANOVA revealed a main effect of Condition, $F(2,144)=4.40, p=.02, \eta^{2}=.06$, as well interactions between Condition and Age Group, $F(6,144)=2.73, p=.02, \eta^{2}=.10$, Condition and Sagittal Plane, $F(4,288)=$ 2.86, $p=.03, \eta^{2}=.04$, and Condition and Coronal Plane, $F(4,288)=4.50, p=.003, \eta^{2}=$. 06. Although the 3-way interaction between Condition, Age Group, and Sagittal or Coronal Plane failed to meet traditional levels of statistical significance, location factors were included in the follow-up analyses given the a priori interest in examining changes in both the magnitude and distribution of the ERP effects. 


\section{Differences between conditions for each age group (800-1000ms)}

In order to investigate whether condition effects were present in all the age groups, followup analyses were conducted separately for each age group using 3 Condition (2-details, 0 details, new) $\times 3$ Coronal Plane (frontal, central, parietal) $\times 3$ Sagittal Plane (left, midline, right) RM ANOVA. For the 3-year-old children, there was a significant interaction between Condition and Coronal Plane, $F(4,68)=3.46, p=.02, \eta^{2}=.17$. Follow-up analyses revealed main effects of Condition at both frontal $(p=.04)$ and parietal $(p=.02)$ planes. At frontal leads, positive amplitude to new items $(M=2.91 \mu \mathrm{V}, S D=3.04)$ was greater than amplitude to old items recalled without contextual details $(.97 \mu \mathrm{V}, S D=2.12)$ with amplitude to old items recalled with contextual details in between $(1.18 \mu \mathrm{V}, S D=3.13)$. A similar pattern emerged at parietal leads, negative amplitude to new items $(-1.46 \mu \mathrm{V}, S D=$ 3.20) was greater than amplitude to old items recalled without contextual details $(.41 \mu \mathrm{V}, S D$ $=2.72)$ with amplitude to old items recalled with contextual details in between $(-.28 \mu \mathrm{V}, S D$ $=2.76$ ).

For the 4-year-old children, there was a marginal main effect of Condition, $F(2,34)=2.75$, $p=.086, \eta^{2}=.14$, and a marginal interaction between Condition and Sagittal Plane, $F(4,68)$ $=2.25, p=.095, \eta^{2}=.12$. Although these effects do not meet conventional levels of significance, follow-up tests were run in order to compare the pattern of responses to the three conditions in the other Age Groups. Similar to the pattern observed in the 3-year-old children, in the left hemisphere $(p=.01)$, average amplitude to new items $(1.59 \mu \mathrm{V}, S D=$ 2.56) was greater than amplitude to old items recalled without contextual details $(-.81 \mu \mathrm{V}$, $S D=2.21)$ with amplitude to old items recalled with contextual details in between $(.97 \mu \mathrm{V}$, $S D=2.81)$.

For the 5-year-old children, there was a marginal interaction between Condition and Coronal Plane, $F(4,88)=2.31, p=.096, \eta^{2}=.10$. Again, although these effects do not meet conventional levels of significance, follow-up tests were run in order to compare the pattern of responses to that observed in the other Age Groups. At central electrodes $(p=.03)$ amplitude to old items recalled without contextual details $(5.12 \mu \mathrm{V}, S D=2.04)$ was greater than amplitude to old items recalled with contextual details $(3.87 \mu \mathrm{V}, S D=1.42)$ with amplitude to new items in between $(4.34 \mu \mathrm{V}, S D=1.90)$, a pattern that differed from that observed in the 3- and 4-year-old children.

For the 6-year-old children, there was a main effect of Condition, $F(2,32)=9.70, p=.001$, $\eta^{2}=.38$, and a significant interaction between Condition, Sagittal Plane, and Coronal Plane, $F(8,128)=2.50, p=.048, \eta^{2}=.14$. Follow-up analyses revealed that in the left hemisphere ( $p=.01)$ amplitude to new items $(2.01 \mu \mathrm{V}, S D=1.98)$ was greater than amplitude to items recalled without contextual details $(-.01 \mu \mathrm{V}, S D=2.70)$ with amplitude to items recalled with contextual details in between $(.81 \mu \mathrm{V}, S D=2.23)$, a pattern similar to that observed in 3- and 4-year-old children. At the midline leads $(p=.076)$, in the frontal plane (Fz) amplitude was marginally greater to items recalled with contextual details $(3.7 \mu \mathrm{V}, S D=4.60)$ than to items recalled without contextual details $(.82 \mu \mathrm{V}, S D=4.41)$, with amplitude to new items in between $(2.5 \mu \mathrm{V}, S D=4.46)$. In contrast, at the parietal plane $(\mathrm{Pz})$ amplitude was marginally greater to items recalled without contextual details $(3.89 \mu \mathrm{V}, S D=5.12)$ than amplitude to 
items recalled with contextual details $(1.47 \mu \mathrm{V}, S D=4.27)$ with amplitude to new items in between $(1.96 \mu \mathrm{V}, S D=2.97)$, a pattern similar to that observed in 5-year-old children. Finally, in the right hemisphere $(p=.04)$ at the central plane (C6) amplitude to items recalled with contextual details $(4.6 \mu \mathrm{V}, S D=2.49)$ and new items $(5.2 \mu \mathrm{V}, S D=2.78)$ were both greater than amplitude to items recalled without contextual details $(1.8 \mu \mathrm{V}, S D=3.32)$ a pattern that differed from all previous Age Groups.

In summary, amplitude to new items was greater than items recalled without contextual details in 3-, 4-, and 6-year-old children, although the location of this effect differed between the age groups. In 5- and 6-year-old children reliable differences began to emerge between items recalled with and without contextual details. Again, the location of these effects differed between the age groups, as did the direction of the effect. In 5-year-old children, amplitude to items recalled without contextual details was greater than amplitude to items recalled with contextual details, whereas in 6-year-old children, amplitude to items recalled with contextual details was greater than amplitude to items recalled without contextual details.

\section{Age-related differences in ERP responses for the two memory conditions (800-1000ms)}

In order to investigate possible age-related changes in neural activity to each of the memory conditions, follow-up analyses were conducted for each condition separately for difference waves for both a) items recalled with contextual details minus new items, and b) items recalled without contextual details minus new items) using a 4 Age Group (3, 4, 5, 6 years) $\times 3$ Coronal Plane (frontal, central, parietal) $\times 3$ Sagittal Plane (left, midline, right) RM ANOVA (see Analytic Approach for justification). Results indicated that adjusted amplitude to items recalled with contextual details showed a marginal interaction between Age Group and Sagittal Plane, $F(6,144)=2.08, p=.065, \eta^{2}=.08$. Follow-up analyses revealed across midline leads $(p=.005)$ adjusted amplitude to items recalled with contextual details was smaller in 3-year-old children $(-1.97$ adjusted $\mu \mathrm{V})$ compared with 4 - (.43 adjusted $\mu \mathrm{V}), 5-($. 52 adjusted $\mu \mathrm{V})$, and 6-year-old children (.51 adjusted $\mu \mathrm{V})$, see Figure $2 \mathrm{~A}$.

Analysis of adjusted amplitude to items recalled without contextual details revealed a significant main effect of Age Group, $F(3,72)=4.35, p=.007, \eta^{2}=.15$. Across all leads, adjusted amplitude to items recalled without contextual details was greater in 5-year-old children (.29 adjusted $\mu \mathrm{V})$ compared to $4-(-.74$ adjusted $\mu \mathrm{V})$, and 6-year-old children $(-1.10$ adjusted $\mu \mathrm{V})$ with 3 -year-old children in between (-.31 adjusted $\mu \mathrm{V})$, see Figure $2 \mathrm{~B}$.

\section{Discussion}

Findings from the present investigation are consistent with previous behavioral research and demonstrate that, across early childhood, children can reliably discriminate old from new items over a 24-48 hour delay, and that memory for details associated with these items improves between 3 and 6 years. Results from analysis of the ERP data contribute novel information regarding the neural mechanisms underlying these effects. Findings revealed that differences in ERP amplitude to the conditions of interest (i.e., items recalled with 
contextual details, items recalled without contextual details) were apparent between 3 to 6 years of age. However, both the pattern of the observed effects and their distribution across the scalp differed as a function of age. Interpreted within a dual-process model of memory framework, these findings suggest age-related differences in neural activity associated with both recollection and familiarity memory processes. Specifically, for items recalled with contextual details, which require recollection, differences were observed between 3-year-old children and all older age groups, whereas for items recalled without contextual details, which index familiarity, differences were observed between 5-year-old children and all other age groups.

Dual process models of memory propose that two separable cognitive processes are associated with successful memory: recollection and familiarity (e.g., Yonelinas, 2002, cf. Malmberg, 2008; Wixted \& Mickes, 2010). Although objective source memory paradigms such as the one utilized in the present study do not produce dependent measures that are "process pure", based on previous literature, in the present investigation we assume that recollection was, by definition, engaged when items were recalled with contextual details (see Cycowicz et al., 2003; Rugg \& Curran, 2007). In contrast, we assume that recollection was not engaged (or perhaps was engaged less often across trials) when items were recalled without contextual details. Using these definitions within the current investigation suggests that neural activity generated by items recalled with contextual details may reflect processes involved in or associated with recollection whereas neural activity generated by items recalled without contextual details reflects processes involved in or associated with familiarity. Under this theoretical framework, findings in the present study suggest agerelated changes in both recollection and familiarity processes.

The finding that children's ability to behaviorally recall contextual details associated with events increases during early childhood is consistent with several previous studies in schoolaged children (Bauer et al., 2012; Cycowicz et al., 2001; Drummey \& Newcombe, 2002; Ghetti et al., 2010; Hamond \& Fivush, 1991; Riggins et al., 2009; Riggins, 2014; Sluzenski et al., 2006; see also, Anooshian, 1999; Anooshian \& Seibert, 1996; Billingsley et al., 2002; Brainerd et al., 2004; Brainerd et al., 2012; Friedman et al., 2010; Lloyd et al., 2009; Ghetti \& Angelini, 2008; Ghetti et al., 2010). However, findings from the present study extend previous work by documenting that these changes are also accompanied by age-related increases in ERP amplitude late in the ERP response (i.e., between 800-1000ms) between 3 and 4 years of age, which suggests that the increase at the behavioral level may be due to increased resources at the neural level. Interestingly, although developmental improvements were present in children's ability to recall contextual details associated with events, even the youngest children were able to reliably recall items with both contextual details as well as accurately discriminate items they encountered during the previous encoding session and those they only encountered during the ERP paradigm. Taken together, these findings suggest that although the ability to remember details associated with events is present in some form by 3 years of age this ability shows protracted development across early childhood. In the present study, the greatest behavioral gains were observed between 3 and 4 years. This is also the period during which changes in neural activity thought to reflect recollection showed significant differences (i.e., the greatest increase in both absolute and 
adjusted amplitudes), perhaps suggesting that the behavioral and neural changes were related.

Neural changes to items recalled without contextual details were also found. Specifically, both absolute and adjusted amplitudes to items recalled without contextual details, which served as an index of familiarity, were greater in 5-year-olds compared to all other age groups. At the behavioral level, differences were apparent in the proportion of items children recalled without contextual details. However, these differences were greatest between 3 and 4 years of age. Thus, it is currently unclear why changes at the neural level were only observed in 5-year-old children. Future studies are truly needed to further examine the behavioral and neural differences observed in this study, how these relate to each other, and what they mean in terms of general memory development.

In contrast to previous studies (Ackerman, 1984; Davidson \& Hoe, 1993; Dirks \& Neisser, 1977; Carroll et al., 1985; Cycowicz, Friedman, \& Duff, 2003; Mandler \& Robinson, 1978; Newcombe et al., 1977; Parkin \& Streete, 1988, Riggins, 2014), we did not find age-related improvements in children's ability to behaviorally recognize old stimuli. This is likely because of the relatively short delay combined with the interactive design of the paradigm, which increased the saliency of the stimuli. It is possible that over longer delays, age-related changes in children's memory for individual items would have been detected.

Developmental improvements in memory are not always uniform (e.g., Riggins, 2014). Non-uniform trajectories are easier to explain if developmental changes are occurring in more than one process associated with the ability as age-related changes in these processes can interact or compound over time in order to produce increased gains at certain periods compared to others. Such co-development of underlying processes may result in the development and refinement of a more differentiated and functionally mature ability over time (i.e., increased functional specialization). The suggestion of increased functional specialization in memory networks is consistent with findings from a recent fMRI study in school-aged children showing that, with age, regions of the medial temporal lobe become increasingly specialized for recollection (Ghetti et al., 2010). Results of this study suggested that 8 -year-old children engaged memory regions such as the hippocampus and posterior parahippocampal gyrus indiscriminately for both recollection and item recognition, whereas 14-year-old children and adults engaged these regions selectively for recollection, and age groups in between showed a transitional pattern (Ghetti et al., 2010).

The present study was modeled after ERP studies in older children and adults, which have been very useful in determining developments in neural mechanisms underlying memory (e.g., Spondrel et al., 2011). However, certain methodological changes were required to adapt this approach for use during early childhood. One of the major changes was that the present investigation utilized a passive viewing paradigm during electrophysiological data collection. Although this is a common approach in infants and young children (see DeBoer et al., 2005; Riggins et al., 2013), it differs from methods used in school-aged children and adults that require active retrieval. Based on previous literature, we assume that ERPs generated during passive viewing reflect the engagement in recollective processes that are necessary for source memory performance even though participants were not given directed 
instructions to recall contextual details. The first line of evidence supporting this assertion comes from research in adults examining incidental retrieval. These studies suggest that episodic memories can be brought to mind without directed search instructions (e.g., Berntsen, 1996) and that the neural correlates of these memories are similar to memories generated by directed search instructions (intentional episodic memories (e.g., Kompus, Eichele, \& Hugdahl, \& Nyberg, 2011). Second, previous research in young children suggests that ERP amplitude is correlated with subsequent performance on source memory tasks, and that greater ERP amplitude reflecting recollection of contextual details is associated with increased behavioral recall of such details, suggesting a positive association between incidental and intentional recollection in children (e.g., Riggins et al., 2009; Riggins et al., 2013). Thus, although children were not instructed to retrieve contextual details during ERP recording, there is support for the idea that ERP responses in the present study reflect recollective processes, albeit incidental, as opposed to intentional.

Although we interpret the present findings in the context of dual process models, there are several caveats to keep in mind. First, due to the young age of the children in this study methodological differences exist between the present report and similar work in adults and older children. For example, familiarity processes were inferred by examining items children were able to identify as old, but were unable to recall the contextual details associated with them. This differs from studies in adults by using either the "Remember/Know" paradigm (in which participants are required to subjectively monitor their memories and give "familiar" judgments) or Receiver Operating Characteristic curves (or ROC curves, in which adults are again required to subjectively monitor their memories and give confidence ratings regarding their responses). However, because young children have difficulty with metacognitive tasks such as these, we elected to use a more objective measure (i.e., lack of contextual details), as an index of familiarity. Implications of this and other methodological differences need to be addressed in future studies. Second, participants were only included in the present report if they provided a sufficient number of ERP trials in each of the conditions of interest. This may have led to an underestimation of age-related differences both in behavioral and neural responses. Finally, examining age-related changes in adjusted amplitude between the two memory conditions of interest rests upon the assumption that general age-related non-memory factors produce additive effects. At present, no data exist at present to support or refute this assumption; further work is needed to address this question and its implications for findings such as those reported here.

Despite these caveats, findings from the present investigation suggest that memory development in early childhood may result from the development of multiple underlying processes. Consideration of these processes is important, as similar performance between age groups on a recognition memory task may mask developmental changes in these underlying processes. Novel paradigms (using behavioral, electrophysiological, or other modern techniques such as fMRI) that allow for the distinction of these memory processes in early childhood are greatly needed and represent an important area for further research. Moreover, future studies should investigate whether similar memory networks are engaged at multiple developmental stages (e.g., infancy and early childhood, see Mecklinger et al., 2011 for findings in school-aged children and adolescents), as well as how long and under what conditions developmental changes are apparent (e.g., Brainerd et al., 2012; Friedman et 
al., 2010). Results from this study represent an initial attempt to begin to distinguish agerelated differences in cognitive and neural processes supporting memory in early childhood, which is an important and necessary element to understanding the development of memory in general.

\section{Acknowledgments}

This research was supported by a grant from the National Institutes of Health (HD-R03-067425) and the Department of Psychology at the University of Maryland, College Park. The authors would like to thank the members of the Neurocognitive Development Lab at the University of Maryland for their contribution to this work and the children who participated in this study. We would also like to acknowledge the contribution of 3 anonymous reviewers for their insightful comments that greatly improved this manuscript. Portions of these data were presented at the following meetings: Association for Psychological Science in Washington, DC, May 2011, Cognitive Development Society in Philadelphia, PA, October 2011, and at the Society for Research in Child Development in Seattle, WA, May 2013.

\section{References}

Ackerman BP. Item-specific and relational encoding effects in children's recall and recognition memory for words. Journal of Experimental Child Psychology. 1984; 37:426-450. doi: 10.1016/0022-0965(84)90069-9.

Anooshian LJ. Understanding age differences in memory: Disentangling conscious and unconscious processes. International Journal of Behavioral Development. 1999; 23:1-17. doi: $10.1080 / 016502599383973$.

Anooshian LJ, Seibert PS. Diversity within spatial cognition: Memory processes underlying place recognition. Applied Cognitive Psychology. 1996; 10:281-299. doi:10.1002/ (SICI)1099-0720(199608)10:4<281::AID-ACP382>3.0.CO;2-8.

Bauer PJ, Doydum AO, Pathman T, Larkina M, Güler OE, Burch M. It's all about location, location, location: children's memory for the "where" of personally experienced events. Journal of Experimental Child Psychology. 2012; 113:510-522. doi:10.1016/j.jecp.2012.06.007. [PubMed: 23010356]

Bauer PJ, Wiebe SA, Carver LJ, Waters JM, Nelson CA. Developments in long-term explicit memory late in the first year of life: behavioral and electrophysiological indices. Psychological science. 2003; 14:629-635. [PubMed: 14629697]

Berntsen D. Involuntary autobiographical memories. Applied Cognitive Psychology. 1996; 10:435454. doi:10.1002/(SICI)1099-0720(199610)10:5<435::AID-ACP408>3.0.CO;2-L.

Billingsley RL, Lou Smith M, Pat McAndrews M. Developmental patterns in priming and familiarity in explicit recollection. Journal of Experimental Child Psychology. 2002; 82:251-277. doi:10.1016/ S0022-0965(02)00007-3. [PubMed: 12093109]

Brainerd CJ, Aydin C, Reyna VF. Development of dual-retrieval processes in recall: Learning, forgetting, and reminiscence. Journal of Memory and Language. 2012; 66:763-788. doi:10.1016/ j.jml.2011.12.002. [PubMed: 22778491]

Brainerd CJ, Holliday RE, Reyna VF. Behavioral measurement of remembering phenomenologies: So simple a child can do it. Child Development. 2004; 75:505-522. doi:10.2307/3696655. [PubMed: 15056202]

Brainerd CJ, Reyna VF, Howe ML. Trichotomous processes in early memory development, aging, and neurocognitive impairment: A unified theory. Psychological Review. 2009; 116:783-832. doi: 10.1037/a0016963. [PubMed: 19839684]

Carroll M, Byrne B, Kirsner K. Autobiographical memory and perceptual learning: A developmental study using picture recognition, naming latency, and perceptual identification. Memory \& Cognition. 1985; 13:273-279. doi:10.3758/BF03197690. [PubMed: 4046828]

Carver LJ, Bauer PJ, Nelson CA. Associations between infant brain activity and recall memory. Developmental Science. 2000; 3:234-246. doi:10.1111/1467-7687.00116. 
Cycowicz YM, Friedman D, Duff M. Pictures and their colors: what do children remember? Journal of Cognitive Neuroscience. 2003; 15:759-768. [PubMed: 12965048]

Cycowicz YM, Friedman D, Snodgrass JG, Duff M. Recognition and source memory for pictures in children and adults. Neuropsychologia. 2001; 39:255-267. [PubMed: 11163604]

Czernochowski D, Mecklinger A, Johansson M, Brinkmann M. Age-related differences in familiarity and recollection: ERP evidence from a recognition memory study in children and young adults. Cognitive, Affective, \& Behavioral Neuroscience. 2005; 5:417-433. doi:10.3758/CABN.5.4.417.

Davidson D, Hoe S. Children's recall and recognition memory for typical and atypical actions in script-based stories. Journal of Experimental Child Psychology. 1993; 55:104-126. doi:10.1006/ jecp.1993.1005.

DeBoer, T.; Scott, LS.; Nelson, CA. Event-related potentials in developmental populations. In: Handy, T., editor. Methodological handbook for research using event-related potentials. The MIT Press; Cambridge, MA: 2005. p. 263-297.

Dirks J, Neisser U. Memory for objects in real scenes: The development of recognition and recall. Journal of Experimental Child Psychology. 1977; 23:315-328. doi: 10.1016/0022-0965(77)90108-4.

Drummey AB, Newcombe NS. Developmental changes in source memory. Developmental Science. 2002; 5:502-513. doi:10.1111/1467-7687.00243.

Friedman D, Johnson R Jr. Event-related potential (ERP) studies of memory encoding and retrieval: a selective review. Microscopy Research and Technique. 2000; 51:6-28. doi: 10.1002/1097-0029(20001001)51:1<6::AID-JEMT2>3.0.CO;2-R. [PubMed: 11002349]

Friedman D, de Chastelaine M, Nessler D, Malcolm B. Changes in familiarity and recollection across the lifespan: An ERP perspective. Brain Research. 2010; 1310:124-141. doi:10.1016/j.brainres. 2009.11.016. [PubMed: 19914220]

Ghetti S, Angelini L. The development of recollection and familiarity in childhood and adolescence: Evidence from the dual-process signal detection model. Child Development. 2008; 79:339-358. doi:10.1111/j.1467-8624.2007.01129.x. [PubMed: 18366427]

Ghetti S, DeMaster DM, Yonelinas AP, Bunge SA. Developmental differences in medial temporal lobe function during memory encoding. The Journal of Neuroscience: The Official Journal of the Society for Neuroscience. 2010; 30:9548-9556. doi:10.1523/JNEUROSCI.3500-09.2010. [PubMed: 20631183]

Haan, MD. Infant EEG and Event-Related Potentials. Taylor \& Francis Group; 2007.

Ille N, Berg P, Scherg M. Artifact correction of the ongoing EEG using spatial filters based on artifact and brain signal topographies. Journal of Clinical Neurophysiology: Official Publication of the American Electroencephalographic Society. 2002; 19:113-124. [PubMed: 11997722]

Lloyd ME, Doydum AO, Newcombe NS. Memory binding in early childhood: Evidence for a retrieval deficit. Child Dvelopment. 2009; 80:1321-1328. doi:10.1111/j.1467-8624.2009.01353.x.

Luck, SJ. An Introduction to the Event-Related Potential Technique (Cognitive Neuroscience). 1. A Bradford Book; 2005.

Lukowski AF, Wiebe SA, Haight JC, DeBoer T, Nelson CA, Bauer PJ. Forming a stable memory representation in the first year of life: Wy imitation is more than child's play. Developmental Sience. 2005; 8:279-298. doi:10.1111/j.1467-7687.2005.00415.x.

Macmillan, NA.; Creelman, CD. Detection theory: A user's guide. Lawrence Erlbaum Associates; 2005.

Malmberg KJ. Recognition memory: A review of the critical findings and an integrated theory for relating them. Cognitive Psychology. 2008; 57:335-384. doi:10.1016/j.cogpsych.2008.02.004. [PubMed: 18485339]

Mandler JM, Robinson CA. Developmental changes in picture recognition. Journal of Experimental Child Psychology. 1978; 26:122-136. doi:10.1016/0022-0965(78)90114-5. [PubMed: 670878]

Marshall DH, Drummey AB, Fox NA, Newcombe NS. An event-related potential study of item recognition memory in children and adults. Journal of Cognition and Development. 2002; 3:201224. doi:10.1207/S15327647JCD0302_4. 
Mecklinger A, Brunnemann N, Kipp K. Two processes for recognition memory in children of early school age: An event-related potential study. Journal of Cognitive Neuroscience. 2011; 23:435446. doi:10.1162/jocn.2010.21455. [PubMed: 20146611]

Nelson, CA. The neurobiological basis of early memory development. In: Cowan, N., editor. The development of memory in childhood. Psychology Press; Hove, UK: 1997. p. 41-82.

Newcombe, NS.; Crowley, SL. To have and have not: What do we mean when we talk about longterm memory development?. In: Oakes, LM.; Bauer, PJ., editors. Short- and long-term memory in infancy and early childhood: Taking the first steps toward remembering. Oxford University Press; 2007.

Newcombe N, Rogoff B, Kagan J. Developmental changes in recognition memory for pictures of objects and scenes. Developmental Psychology. 1977; 13:337-341. doi: 10.1037/0012-1649.13.4.337.

Parkin AJ, Streete S. Implicit and explicit memory in young children and adults. British Journal of Psychology. 1988; 79:361-369. doi:10.1111/j.2044-8295.1988.tb02295.x.

Reynolds GD, Richards JE. Familiarization, attention, and recognition memory in infancy: A eventrelated potential and cortical source localization study. Developmental Psychology. 2005; 41:598615. doi:10.1037/0012-1649.41.4.598. [PubMed: 16060807]

Riggins, T. Methodological Handbook for Research Using Event-related Potentials. MIT Press; Cambridge, MA: 2005. Event-related potentials in developmental populations; p. 263-297.

Riggins, T. Origins and Development of Recollection: Perspectives from Psychology and Neuroscience. Oxford University Press; 2012. Building blocks of recollection; p. 42-72.

Riggins T. Longitudinal investigation of source memory reveals qualitative differences between item memory and binding. Developmental Psychology. 2014; 50:449-459. [PubMed: 23855256]

Riggins T, Miller NC, Bauer PJ, Georgieff MK, Nelson CA. Electrophysiological indices of memory for temporal order in early childhood: Iplications for the development of recollection. Developmental Science. 2009a; 12:209-219. doi:10.1111/j.1467-7687.2008.00757.x. [PubMed: 19143795]

Riggins T, Miller NC, Bauer PJ, Georgieff MK, Nelson CA. Consequences of Low Neonatal Iron Status due to Maternal Diabetes Mellitus on Explicit Memory Performance in Childhood. Developmental Neuropsychology. 2009b; 34:762-779. doi:10.1080/87565640903265145. [PubMed: 20183732]

Riggins T, Rollins L, Graham M. Electrophysiological investigation of source memory in early childhood. Developmental Neuropsychology. 2013; 38:180-196. doi: 10.1080/87565641.2012.762001. [PubMed: 23573796]

Rollins L, Riggins T. Developmental changes in memory encoding: Insights from event-related potentials. Developmental Science. 2013; 16:599-609. doi:10.1111/desc.12072. [PubMed: 23786477]

Rose SA, Feldman JF, Jankowski JJ, Van Rossem R. The structure of memory in infants and toddlers: An SEM study with full-terms and preterms. Developmental Science. 2011; 14:83-91. doi: 10.1111/j.1467-7687.2010.00959.x. [PubMed: 21159090]

Rovee-Collier C. Dissociations in infant memory: Rethinking the development of implicit and explicit memory. Psychological Review. 1997; 104:467-498. [PubMed: 9243961]

Rugg MD, Curran T. Event-related potentials and recognition memory. Trends in Cognitive Sciences. 2007; 11(6):251-257. doi:10.1016/j.tics.2007.04.004. [PubMed: 17481940]

Sprondel V, Kipp KH, Mecklinger A. Developmental changes in item and source memory: Evidence from an ERP recognition memory study with children, adolescents, and adults. Child Dvelopment. 2011; 82:1638-1953. doi:10.1111/j.1467-8624.2011.01642.x.

Squire LR, Wixted JT, Clark RE. Recognition memory and the medial temporal lobe: A new perspective. Nature Reviews Neuroscience. 2007; 8:872-883.

Yonelinas AP. The nature of recollection and familiarity: A review of 30 years of research. Journal of Memory and Language. 2002; 46:441-517. doi:10.1006/jmla.2002.2864.

Wixted JT, Mickes L. A continuous dual-process model of remember/know judgments. Psychological Review. 2010; 117:1025-1054. [PubMed: 20836613] 

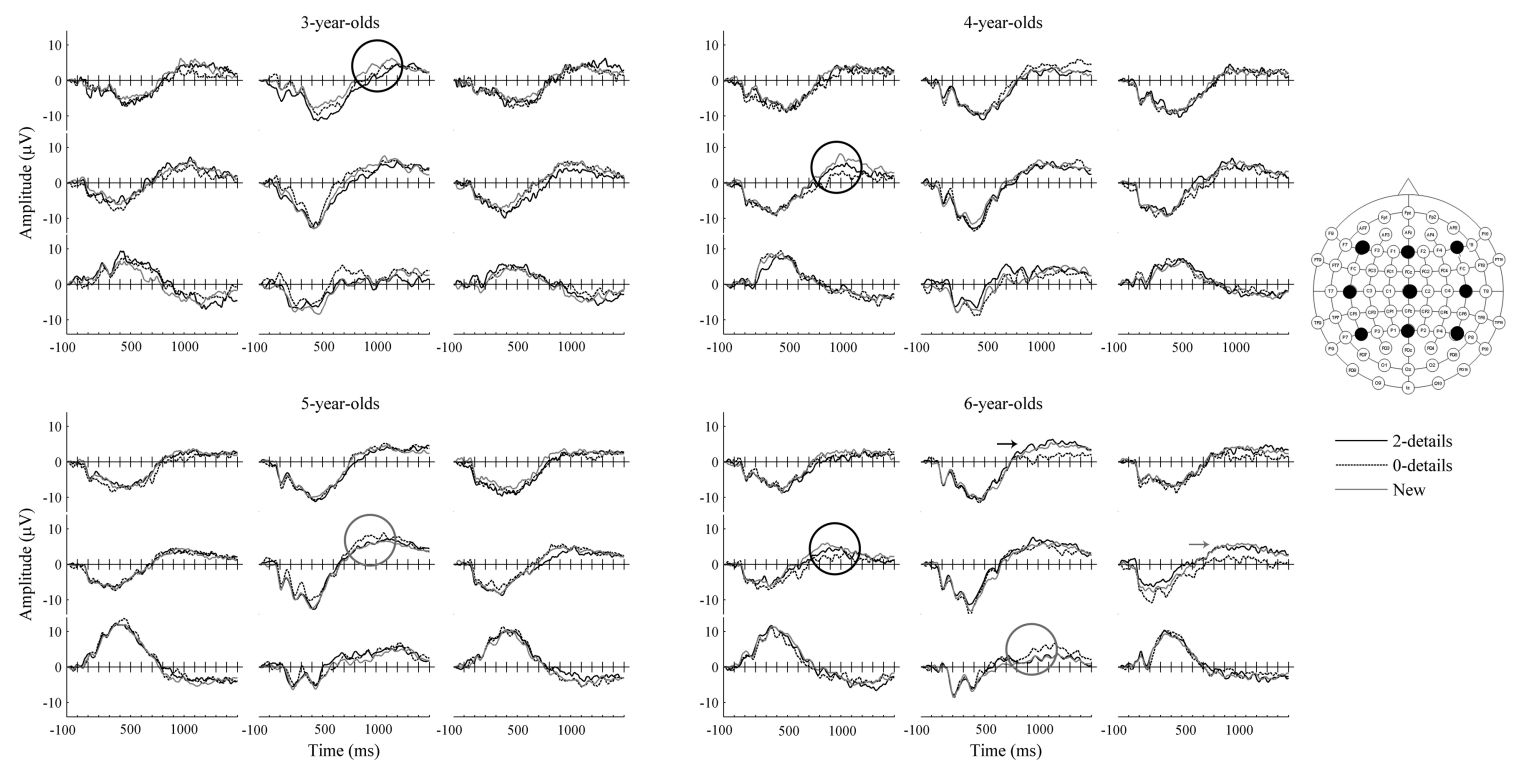

Figure 1.

Average ERP responses to items recalled with contextual details, items recalled without contextual details, and new items for 3-, 4-, 5- and 6-year-old children. Displayed leads from left to right/top to bottom are: F5, Fz, F6, C5, Cz, C6, and P5, Pz, P6. Colored circles illustrate differences between conditions that were similar across age groups (although in different locations). Arrows illustrate differences between conditions that were only apparent in the 6-year-old age group. 
A
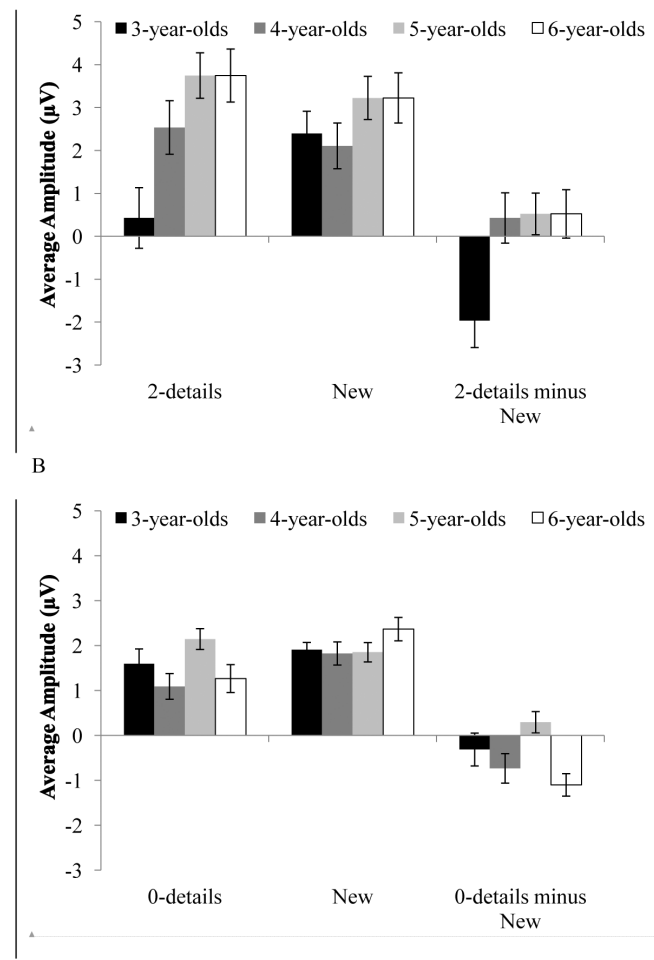

Figure 2.

Average amplitude across midline leads for items recalled with contextual details, new items, and the difference between these conditions plotted as a function of age group (A). Average amplitude across all leads for items recalled without contextual details, new items, and the difference between these conditions plotted as a function of age group (B). 


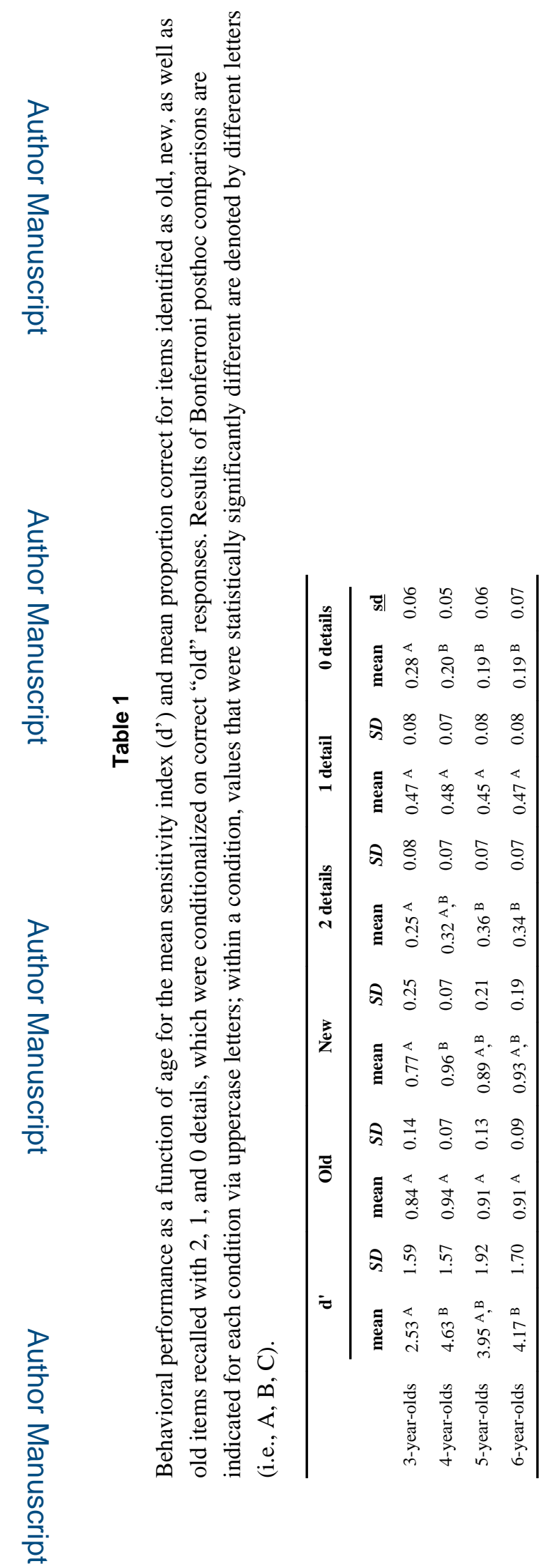

Child Dev. Author manuscript; available in PMC 2016 May 01. 